\title{
Real-World Experience of Nivolumab in Non-small Cell Lung Cancer in Korea
}

\section{Sun Min Lim, MD, PhD ${ }^{1}$ \\ Sang-We Kim, MD, PhD² \\ Byoung Chul Cho, MD, PhD ${ }^{1}$ \\ Jin Hyung Kang, MD, PhD \\ Myung-Ju Ahn, MD, PhD 4 \\ Dong-Wan Kim, MD, PhD \\ Young-Chul Kim, MD, PhD \\ Jin Soo Lee, MD, PhD ${ }^{7}$ \\ Jong-Seok Lee, MD, $\mathrm{PhD}^{8}$ \\ Sung Yong Lee, MD, $\mathrm{PhD}^{9}$ \\ Keon Uk Park, MD, PhD'10 \\ Ho Jung An, MD, PhD'11 \\ Eun Kyung Cho, MD, PhD ${ }^{12}$ \\ Tae Won Jang, MD, $P h D^{13}$ \\ Bong-Seog Kim, MD, PhD ${ }^{14}$ \\ Joo-Hang Kim, MD, PhD ${ }^{15}$ \\ Sung Sook Lee, MD, PhD ${ }^{16}$ \\ Im-II Na, MD, PhD'77 \\ Seung Soo Yoo, MD, PhD 18 \\ Ki Hyeong Lee, MD, PhD'19}

*A list of author's affiliations appears at the end of the paper.

\author{
Correspondence: Ki Hyeong Lee, MD, PhD \\ Division of Hematology-Oncology, \\ Department of Internal Medicine, \\ Chungbuk National University Hospital, \\ Chungbuk National University College of \\ Medicine, 776 ilsunhwan-ro, Heungdeok-gu, \\ Cheongju 28644, Korea \\ Tel: 82-43-269-6015 \\ Fax: 82-43-269-6899 \\ E-mail: kihlee@chungbuk.ac.kr \\ Received March 24, 2020 \\ Accepted May 9, 2020 \\ Published Online May 15, 2020
}

\section{Purpose}

The introduction of immune checkpoint inhibitors represents a major advance in the treatment of lung cancer, allowing sustained recovery in a significant proportion of patients. Nivolumab is a monoclonal anti-programmed death cell protein 1 antibody licensed for the treatment of locally advanced or metastatic non-small cell lung cancer (NSCLC) after prior chemotherapy. In this study, we describe the demographic and clinical outcomes of patients with advanced NSCLC treated with nivolumab in the Korean expanded access program.

\begin{abstract}
Materials and Methods
Previously treated patients with advanced nonsquamous and squamous NSCLC patients received nivolumab at $3 \mathrm{mg} / \mathrm{kg}$ every 2 weeks up to 36 months. Efficacy data including investigator-assessed tumor response, progression data, survival, and safety data were collected.
\end{abstract}

\section{Results}

Two hundred ninety-nine patients were treated across 36 Korean centers. The objective response rate and disease control rate were $18 \%$ and $49 \%$, respectively; the median progression-free survival was 2.1 months (95\% confidence interval [Cl], 1.87 to 3.45), and the overall survival (OS) was 13.2 months ( $95 \% \mathrm{Cl}, 10.6$ to 18.9). Patients with smoking history and patients who experienced immune-related adverse events showed a prolonged OS. Cox regression analysis identified smoking history, presence of immune-related adverse events as positive factors associated with OS, while liver metastasis was a negative factor associated with OS. The safety profile was generally comparable to previously reported data.

\section{Conclusion}

This real-world analysis supports the use of nivolumab for pretreated NSCLC patients, including those with an older age.

\section{Key words}

Non-small cell lung cancer, Anti-PD-1, Real-world data

\section{Introduction}

The introduction of immune checkpoint inhibitors (ICIs) has led to tremendous changes in the treatment of advanced stage non-small cell lung cancer (NSCLC), and ICIs have emerged as one of the most effective anticancer agents. ICIs can block inhibitory pathways that control the immune response, restoring and sustaining the immune system against cancer cells. Programmed death cell protein 1 (PD-1) is a promising target of immunotherapy, and tumor expression of 
programmed death-ligand 1 (PD-L1) has been widely investigated as a predictive marker of response, although its sensitivity and specificity is modest [1]. Recent pivotal studies have assessed the role of immunotherapy in metastatic NSCLCs in both squamous and nonsquamous histology, and three agents (nivolumab, pembrolizumab, atezolizumab) have been investigated for the treatment of previously treated metastatic NSCLC.

Nivolumab is a human IgG4 monoclonal antibody that blocks PD-1, and is now approved in second-line therapy of metastatic NSCLC patients. Nivolumab was tested in the open label, randomized phase 3 trials of CheckMate 017 and $057[2,3]$ for previously treated squamous and nonsquamous NSCLC, respectively, and showed significantly improved OS compared to docetaxel in both trials. Recently, 5-year pooled OS rates for CheckMate 017 and CheckMate057 were reported to be $13.4 \%$ for nivolumab, whereas it was $2.6 \%$ for docetaxel [4]. However, these clinical trials have excluded patients with poor performance status, brain metastases, and epidermal growth factor receptor $(E G F R)$ / anaplastic lymphoma kinase $(A L K)$ genomic alterations. About one-third of lung cancer patients present with poor performance status (Eastern Cooperative Oncology Group performance status [ECOG PS] $\geq 2$ ) in the real world, although they have been excluded from most clinical trials [5]. While there is a higher frequency of EGFR and $A L K$-altered patients in Asia, this subgroup of patients cannot be overlooked in the era of immunotherapy. Moreover, elderly patients are frequently under-represented in clinical trials, despite the growing population worldwide [6].

Nivolumab was provided by Ono Pharmaceuticals through an expanded access program (EAP) from February 2016 to March 2019 for both squamous and nonsquamous NSCLC patients in Korea. The EAP program enrolled 300 advanced NSCLC patients from 36 sites in Korea, and represents the largest nation-wide representation of real-world practice. Here, we present the characteristics of response and toxicity of nivolumab treatment in multiple centers in Korea.

\section{Materials and Methods}

\section{Patients and data collection}

Advanced NSCLC patients were screened and recruited from 36 academic hospitals across the Republic of Korea. Eligibility criteria included locally advanced or metastatic NSCLC that had progressed despite standard therapy, an ECOG PS of 0 to 2, and adequate organ function and laboratory results. Key exclusion criteria included active brain metastases, autoimmune diseases, and patients with a life expectancy of $<6$ weeks. Nivolumab was given $3 \mathrm{mg} / \mathrm{kg}$ intravenously every 2 weeks for a maximum of 36 months or until disease progression, unacceptable toxicity, or withdrawal of consent. Dose escalation or reduction was not allowed.

\section{Efficacy assessment}

Baseline tumor assessment was performed before the start of treatment, and response evaluation was performed by computed tomography imaging at least every 3 months, according to the local standard of practice. Tumor size measurement was performed according to RECIST 1.1 criteria [7]. An overall response was defined as a complete response (CR) or partial response (PR). Other efficacy parameters included disease control rate, duration of response, progression-free survival (PFS), and overall survival (OS). PFS was defined as the time from the start of nivolumab treatment to disease progression or death from any cause. OS was defined as the time from the start of nivolumab treatment to death from any cause.

\section{Safety assessment}

Safety was assessed at each patient visit by routine physical examination and laboratory assessment as needed by the physician. Blood tests included hematology, routine chemistry (including liver, kidney function, and pancreatic enzymes) and hormonal measurements (thyroid, adrenal function). Toxicity was classified according to Common Terminology Criteria for Adverse Events ver. 4 (CTCAE v4.0), and data regarding immune-related adverse events (irAEs) were reported retrospectively by patient chart review and laboratory reports.

\section{Statistical analysis}

All patients who received at least one dose of nivolumab were included in the intention-to-treat analyses for efficacy and safety. Data were summarized using descriptive statistics or contingency tables for demographic and baseline characteristics, response measurements, and safety measurements. All survival analyses were estimated using KaplanMeier curves and compared using the log-rank test. Hazard ratios and corresponding confidence intervals were estimated with the Cox proportional hazards model. All statistical analyses were performed with SPSS ver. 25.0 (IBM Corp., Armonk, NY).

\section{Ethical statement}

This program was performed in accordance with the principles of the Good Clinical Practice and was approved by the institutional review board of each hospital. All patients provided written informed consent before participation in the EAP.

\section{Results}

\section{Baseline characteristics}

From February 2016 to September 2016, a total of 334 patients were screened and 300 patients were enrolled in the 


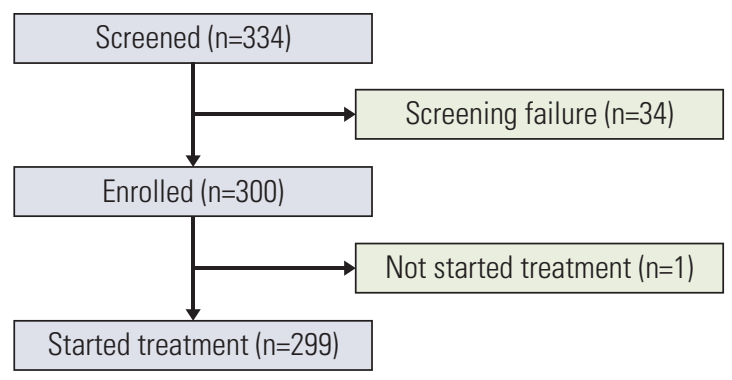

Fig. 1. Flow diagram of the study patients. Three hundred thirty-four patients were nominated for treatment in the nivolumab EAP. Thirty-four patients did not meet the study criteria and failed the screening. A total of 300 patients were enrolled, but one patient did not start the treatment. Overall, 299 patients were included in the analysis.

EAP. One patient did not start the treatment, so a total of 299 patients were evaluated for intention-to-treat analysis (Fig. 1). Median follow up time was 30.1 months (95\% confidence interval [CI], 0 to 36.3), and a median of 6 doses of nivolum$a b$ were administered (range, 1 to 79). The median age of all patients was 61 (range, 31 to 85 years), and $206(68.9 \%)$ were male patients. Most patients (87.3\%) had ECOG PS 0-1, but 38 patients (12.7\%) had ECOG PS 2. By histology, 198 (66.2\%) patients had adenocarcinoma, $85(28.4 \%)$ had squamous cell carcinoma, 6 (2\%) had large cell carcinoma. Distant metastasis was identified in 275 (91.9\%) patients, and the most common site of metastasis was bone $(27.1 \%)$, followed by lung $(24.4 \%)$ and brain (20.1\%). Regarding smoking history, 108 (36.1\%) patients were never-smokers, and former or current smokers (63.9\%) were more prevalent. Most patients (86.9\%) had stage IV disease and were former or current smokers $(63.9 \%)$. As previous therapy, $61.5 \%$ of patients had surgery, $51.8 \%$ had radiotherapy, and $27.1 \%$ had received one line of chemotherapy before nivolumab. The majority of patients $(72.9 \%)$ had received two or more lines of chemotherapy, ranging from 2 to 7 (Table 1).

As PD-L1 testing was optional in this program, PD-L1 immunohistochemistry results were available in only 17 patients (5.7\%), EGFR mutations were identified in 48 patients (16.1\%), and $A L K$ translocations were identified in five patients (1.7\%), but EGFR and ALK gene status was not available in $155(51.8 \%)$ and 176 (58.9\%) patients, respectively.

\section{Efficacy}

Response evaluation was available in 256 patients, and 43 patients (14\%) had missing evaluation scans due to progressive disease or death before first evaluation (Table 2). Best objective overall response (ORR) in the evaluable population was: CR in four patients (2\%), PR in 49 patients (16\%), stable disease in 92 patients (31\%), and progressive disease in $111(37 \%)$ patients. The ORR was $18 \%$, and disease control rate (DCR) was $49 \%$. The median time to response was 1.8
Table 1. Baseline characteristics of all patients

\begin{tabular}{|c|c|}
\hline Characteristic & No. $(\%)(n=299)$ \\
\hline \multicolumn{2}{|l|}{ Sex } \\
\hline Male & $206(68.9)$ \\
\hline Female & $93(31.1)$ \\
\hline \multicolumn{2}{|l|}{ Age (yr) } \\
\hline Median (range) & $61(31-85)$ \\
\hline \multicolumn{2}{|l|}{ ECOG PS } \\
\hline $0-1$ & $261(87.3)$ \\
\hline 2 & 38 (12.7) \\
\hline \multicolumn{2}{|l|}{ Histology } \\
\hline Adenocarcinoma & $198(66.2)$ \\
\hline Squamous cell carcinoma & $85(28.4)$ \\
\hline Large cell carcinoma & $6(2.0)$ \\
\hline Other & $10(3.3)$ \\
\hline \multicolumn{2}{|l|}{ Metastasis site } \\
\hline Adrenal glands & $31(10.4)$ \\
\hline Bone & $81(27.1)$ \\
\hline Brain & $60(20.1)$ \\
\hline Liver & $32(10.7)$ \\
\hline Lung ipsilateral & $55(18.4)$ \\
\hline Lung contralateral & $73(24.4)$ \\
\hline Other & $109(36.5)$ \\
\hline \multicolumn{2}{|l|}{ Clinical stage } \\
\hline IIIA & $2(0.7)$ \\
\hline IIIB & $37(12.4)$ \\
\hline IV & $260(87.0)$ \\
\hline \multicolumn{2}{|l|}{ Smoking history } \\
\hline Never & $108(36.1)$ \\
\hline Former & $171(57.2)$ \\
\hline Current & $20(6.7)$ \\
\hline \multicolumn{2}{|l|}{ Previous therapy } \\
\hline \multicolumn{2}{|l|}{ Surgery } \\
\hline Yes & 115 (38.5) \\
\hline No & $184(61.5)$ \\
\hline \multicolumn{2}{|l|}{ Radiotherapy } \\
\hline Yes & $144(48.2)$ \\
\hline No & $155(51.8)$ \\
\hline \multicolumn{2}{|l|}{ Chemotherapy } \\
\hline 1 & $81(27.1)$ \\
\hline$\geq 2$ & $218(72.9)$ \\
\hline
\end{tabular}

ECOG PS, Eastern Cooperative Oncology Group performance status.

months (range, 1.3 to 18.2 months), and the median duration of response in those who achieved objective response was 21.0 months (range, $0.8+$ to $33.2+$ months). We compared ORR according to histology (squamous cell carcinoma vs. adenocarcinoma) and smoking status (never vs. former / current). The ORR ( $24.7 \%$ vs. $13.6 \%, \mathrm{p}=0.023)$ and DCR $(56.5 \%$ vs. $42.9 \%, \mathrm{p}=0.036)$ in squamous cell carcinoma patients were both significantly higher than adenocarcinoma patients, while the ORR and DCR did not differ between never- 
Table 2. Overall objective response

\begin{tabular}{|c|c|c|c|c|c|}
\hline & \multirow{2}{*}{$\begin{array}{c}\text { Total } \\
(n=299)\end{array}$} & \multicolumn{2}{|c|}{ Histology } & \multicolumn{2}{|c|}{ Smoking } \\
\hline & & $\begin{array}{l}\text { Squamous } \\
\qquad(\mathrm{n}=85)\end{array}$ & $\begin{array}{l}\text { Adenocarcinoma } \\
\qquad(n=198)\end{array}$ & $\begin{array}{l}\text { Never } \\
(n=108)\end{array}$ & $\begin{array}{c}\text { Former or } \\
\text { current }(n=191)\end{array}$ \\
\hline Objective response rate ra) $^{a}$ & $53(18)$ & $21(25)$ & $27(14)$ & $13(12)$ & $40(21)$ \\
\hline Disease control rate ${ }^{\text {b) }}$ & $145(49)$ & $48(56)$ & $85(43)$ & $45(42)$ & $100(52)$ \\
\hline \multicolumn{6}{|l|}{ Best overall response } \\
\hline CR & $4(2)$ & $2(2)$ & $2(1)$ & $1(1)$ & $3(2)$ \\
\hline PR & $49(16)$ & $19(22)$ & $25(13)$ & $12(11)$ & $37(19)$ \\
\hline SD & $92(31)$ & $27(32)$ & $58(29)$ & $32(30)$ & $60(31)$ \\
\hline PD & $111(37)$ & $28(33)$ & $79(40)$ & $43(40)$ & $68(36)$ \\
\hline NE & $43(14)$ & $1(1)$ & 0 & 0 & $1(1)$ \\
\hline Duration of response ${ }^{\mathrm{c})}(\mathrm{mo})$ & $\begin{array}{c}21.03 \\
(0.79+\text { to } 33.15+)\end{array}$ & $\begin{array}{c}16.9 \\
\text { (1.94 to } 33.08+)\end{array}$ & $\begin{array}{c}26.8 \\
(0.79+\text { to } 32.85+)\end{array}$ & $\begin{array}{c}20.4 \\
(2.43 \text { to } 32.39+)\end{array}$ & $\begin{array}{c}26.8 \\
(0.79+\text { to } 33.15+)\end{array}$ \\
\hline
\end{tabular}

Values are presented as number (\%) or median (range). CR, complete response; PR, partial response; SD, stable disease; PD, progressive disease; NE, not evaluable. ${ }^{a}$ Best overall response is $\mathrm{CR}$ or PR, ${ }^{\mathrm{b})}$ Best overall response is $\mathrm{CR}$ or PR or SD, ${ }^{\mathrm{c}}$ The duration of response was defined as the time from the date of first response (CR/PR) to the date of first documented disease progression/ death (event), or last tumor assessment (censored).

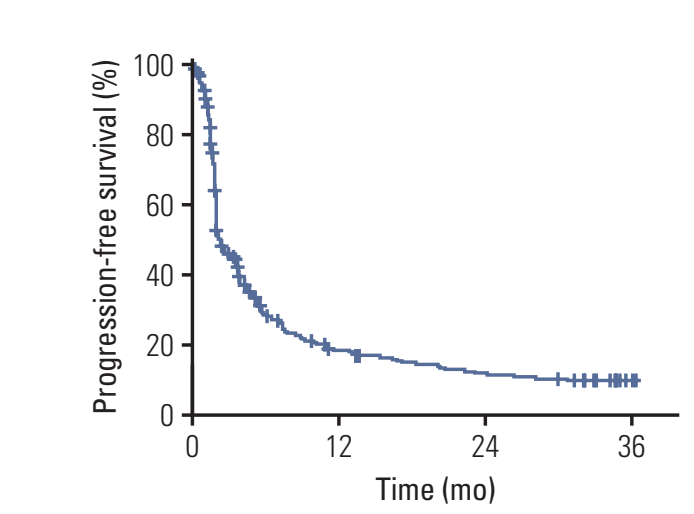

No. at risk 299
A

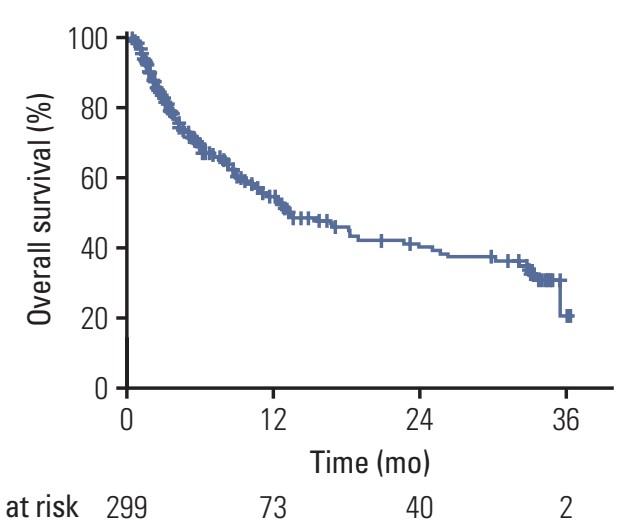

Fig. 2. Kaplan-Meier curves of progression-free survival and overall survival in non-small cell lung cancer patients treated with nivolumab. (A) Progression-free survival of all patients. (B) Overall survival of all patients.

smokers and former/current smokers. The Kaplan-Meier estimates for PFS and OS are reported in Fig. 2A and B. The median PFS was 2.1 months (95\% CI, 1.87 to 3.45), and the median OS was 13.2 months ( $95 \%$ CI, 10.6 to 18.9). The 1-year and 2-year PFS rate was $18.2 \%$ and $11.7 \%$ and, 1-year and 2-year OS rate was $54.5 \%, 40.1 \%$, respectively. Next, PFS and OS were compared between specific patient subgroups. Former or current smokers showed significantly longer OS, but not PFS, compared to never-smokers (Fig. 3A and B). PFS and $\mathrm{OS}$ were not significantly different according to tumor histology (Fig. 3C and D).

\section{Efficacy in specific patients' subgroups}

We compared ORR according to different clinical parameters including metastatic site, ECOG PS, prior treatment line, presence of immune-related $\mathrm{AE}$ (irAE), and EGFR mutation status (S1 Table). We noted a significantly higher ORR in patients who presented with irAE than those who did not $(32 \%$ vs. $11 \%, \mathrm{p}<0.001)$. In addition, patients who received one prior therapy showed higher ORR than those who received two or more prior therapies (27\% vs. $14 \%$, $\mathrm{p}=0.009$ ). However, there were no significant differences in ORR according site of metastasis, ECOG PS or EGFR mutation status.

When PFS and OS were compared between specific patient subgroups, we noted that patients who were aged 75 or older showed a significantly prolonged PFS $(\mathrm{p}=0.046)$ compared to patients under 75 years (S2A Fig.), while OS was not different (S2B Fig.). There were no differences in PFS or OS according to ECOG PS (S2C and S2D Fig.). Patients who experienced irAEs showed a significantly prolonged PFS and OS compared to those who did not $(\mathrm{p}<0.001)$ (S2E and S2F Fig.). 

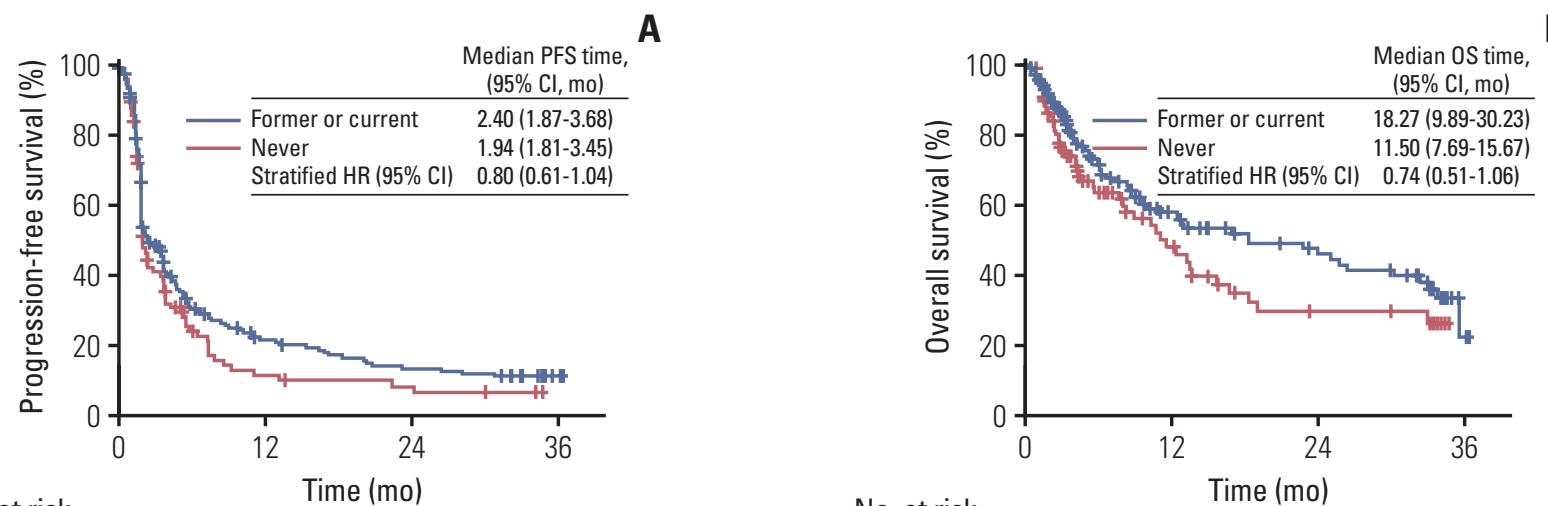

$\begin{array}{rrrrr}\text { No. at risk } & & & & \\ \text { Former or current } & 191 & 30 & 18 & 2 \\ \text { Never } & 108 & 8 & 5 & 0\end{array}$

\begin{tabular}{rrrrr} 
No. at risk & & \multicolumn{3}{c}{ Time $(\mathrm{mo})$} \\
Former or current & 191 & 49 & 30 & 2 \\
Never & 108 & 24 & 10 & 0
\end{tabular}
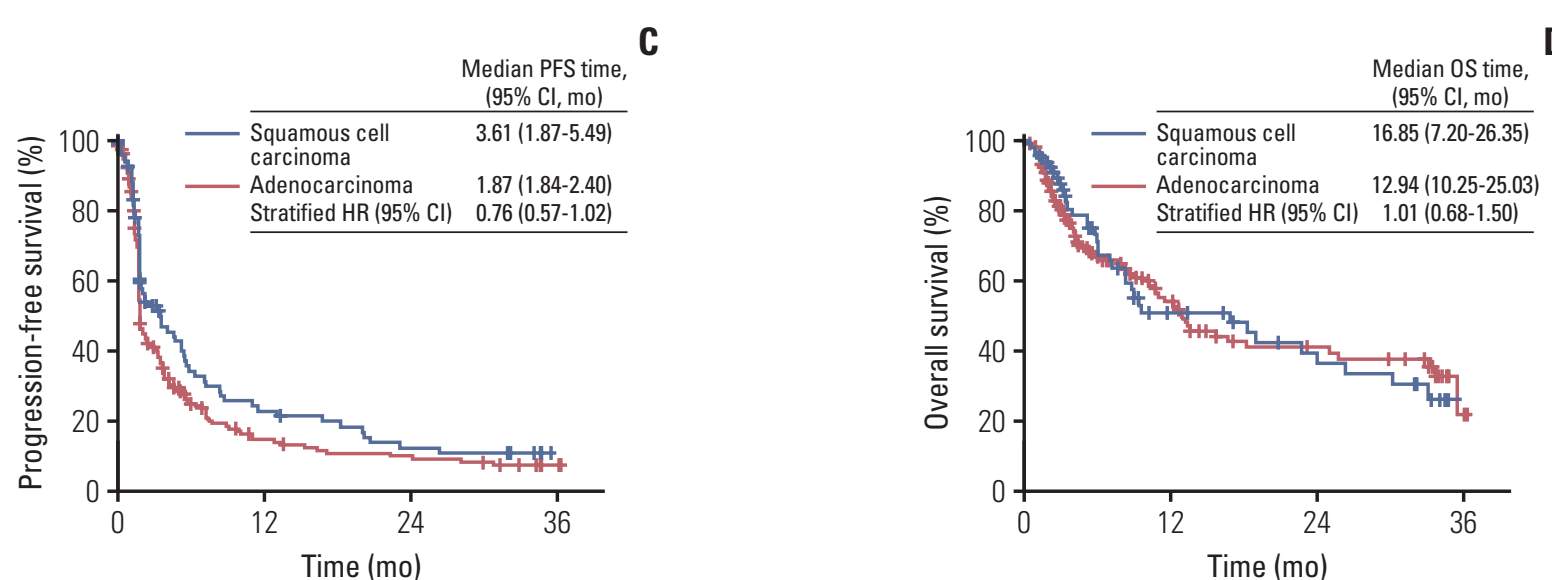

No. at risk

$\begin{array}{rrrrr}\begin{array}{r}\text { Squamous cell } \\ \text { carcinoma }\end{array} & 85 & 16 & 8 & 0 \\ \text { Adenocarcinoma } & 198 & 19 & 12 & 2\end{array}$

No. at risk

$\begin{array}{rrrrr}\begin{array}{r}\text { Squamous cell } \\ \text { carcinoma }\end{array} & 85 & 21 & 12 & 0 \\ \begin{array}{r}\text { Adenocarcinoma } \\ \text { Ader }\end{array} & 46 & 24 & 2\end{array}$

Fig. 3. Kaplan-Meier curves. (A) Comparison of progression-free survival between former or current smokers versus never-smokers. (B) Comparison of overall survival between former or current smokers versus never-smokers. (C) Comparison of progression-free survival between squamous cell carcinoma and adenocarcinoma. (D) Comparison of overall survival between squamous cell carcinoma and adenocarcinoma. HR, hazard ratio; CI, confidence interval.

On the other hand, patients who presented with concomitant brain and liver metastases showed the shortest PFS and OS compared to those with brain, liver, or other metastases (data not shown).

Ninety-three patients were treated beyond progression, and of these patients, two $(2 \%)$ achieved objective response beyond progression, and disease control was achieved in $18(19 \%)$ patients. The median OS of patients who received nivolumab beyond PD was 13.2 months (95\% CI, 9.59 to 23.95), which was significantly longer from those who withdrew after PD (8.28 months; 95\% CI, 6.05 to 12.35; $\mathrm{p}=0.048$ ).

When we compared clinical or tumor characteristics between early progressors ( $<4$ cycles) and long-term responders ( $\geq 48$ cycles), no baseline clinical or tumor characteristics clearly distinguished long-term survivors (data not shown).
There was a trend toward long-term efficacy in patients with squamous histology and patients with smoking history.

\section{Univariate and multivariate analyses}

We next performed univariate and multivariate analyses to assess the role of each clinical parameter on OS and PFS. Cox regression analysis identified smoking history, presence of irAE as positive factors associated with OS, and liver metastasis was negative factor associated with OS. At multivariate analysis, all three factors maintained their independent prognostic role (Table 3). In addition, the presence of irAEs was also a positive factor associated with PFS (S3 Table). 
Table 3. Cox proportional hazard model for overall survival

\begin{tabular}{|c|c|c|c|c|c|c|c|}
\hline \multirow{2}{*}{ Variable } & \multirow{2}{*}{ Reference } & \multicolumn{3}{|c|}{ Univariate analysis } & \multicolumn{3}{|c|}{ Multivariate analysis } \\
\hline & & Hazard ratio & $95 \% \mathrm{CI}$ & p-value & Hazard ratio & $95 \% \mathrm{CI}$ & p-value \\
\hline \multicolumn{8}{|l|}{ Age } \\
\hline$\geq 75$ yr & $<75 \mathrm{yr}$ & 0.62 & $0.31-1.23$ & 0.172 & - & - & - \\
\hline \multicolumn{8}{|l|}{ Smoking history } \\
\hline Former or current & Never & 0.66 & $0.46-0.96$ & 0.028 & 0.65 & $0.44-0.94$ & 0.024 \\
\hline \multicolumn{8}{|l|}{ Histology } \\
\hline Squamous cell carcinoma & Adenocarcinoma & 0.92 & $0.62-1.38$ & 0.697 & - & - & - \\
\hline \multicolumn{8}{|l|}{ Brain metastasis } \\
\hline Yes & No & 1.40 & $0.93-2.11$ & 0.110 & - & - & - \\
\hline \multicolumn{8}{|l|}{ Liver metastasis } \\
\hline Yes & No & 2.38 & $1.52-3.75$ & $<0.001$ & 2.18 & $1.37-3.47$ & 0.001 \\
\hline \multicolumn{8}{|l|}{ ECOG PS } \\
\hline 2 & $0-1$ & 1.60 & $0.97-2.65$ & 0.067 & - & - & - \\
\hline \multicolumn{8}{|l|}{ Previous treatment line } \\
\hline$\geq 2$ & 1 & 1.42 & $0.94-2.15$ & 0.094 & - & - & - \\
\hline \multicolumn{8}{|l|}{ Immune-related $\mathrm{AE}$} \\
\hline Yes & No & 0.44 & $0.29-0.67$ & $<0.001$ & 0.50 & $0.33-0.76$ & 0.001 \\
\hline \multicolumn{8}{|l|}{$\mathrm{AE}$} \\
\hline Yes & No & 0.77 & $0.44-1.35$ & 0.360 & - & - & - \\
\hline \multicolumn{8}{|l|}{ EGFR } \\
\hline Positive & Negative & 0.94 & $0.52-1.72$ & 0.854 & - & - & - \\
\hline
\end{tabular}

CI, confidence interval; ECOG PS, Eastern Cooperative Oncology Group performance status; AE, adverse effect; EGFR, epidermal growth factor receptor.

\section{Safety}

Treatment-related $\mathrm{AE}$ of any grade, and treatment-related AE of grade 3-4 events were reported in $63 \%$ and $18 \%$ of patients, respectively. The discontinuation rate due to treatment-related AEs was 8\%. No treatment-related deaths were reported. IrAEs were reported in $32 \%$ of patients (S4 Table). The most common treatment-related AEs were decreased appetite $(9.7 \%)$, pruritus $(8.0 \%)$, pneumonia $(7.0 \%)$, fatigue $(7.0 \%)$ and diarrhea $(7.0 \%)$. The most common treatmentrelated grade 3-4 AEs were dyspnea (1.7\%), hepatotoxicity $(1.3 \%)$, and pleural effusion (1\%) (S5 Table). The most common irAEs was skin toxicity, occurring in $11 \%$ of patients, followed by endocrine ( $8 \%$ ) and gastrointestinal $(7 \%)$ (S6 Table). The following treatment-related grade 3/4 irAEs were notable: skin toxicity presenting as erythematous skin $\operatorname{rash}(\mathrm{n}=1)$ and rash acneiform $(\mathrm{n}=1)$, pulmonary toxicity presenting as pneumonitis $(\mathrm{n}=2)$, hepatotoxicity presenting as aspartate aminotransferase/alanine transaminase increased $(n=2)$, musculoskeletal toxicity presenting as myalgia $(n=1)$, and endocrine toxicity presenting as thyroid-stimulating hormone increase $(\mathrm{n}=1)$.

\section{Discussion}

In this real-world analysis, efficacy and safety of nivolumab were comparable to previous phase 3 results. ORR, PFS, and OS in our population were similar to the observations in the CheckMate017 and CheckMate057 studies [2,3]. In addition, 3-year OS was $20.4 \%$ in our study, while it was $17.1 \%$ in the pooled phase 3 analyses [4]. There were no new safety signals identified in our study. Unlike clinical trials, we included patients with ECOG PS 2 and those who were previously heavily treated, thus this EAP represents a sizeable real-world experience with nivolumab.

Subgroup analyses showed that patients who experienced irAEs showed significantly higher ORR, PFS, and OS. The correlation between irAEs and efficacy has been previously reported [8-12]. In a recent study conducted in Spain, the probability of having a clinical response was 23 times higher in those patients who showed an irAE [8]. In a series of cases from clinical trials at MD Anderson, patients who were treated with ICIs showed better ORR and PFS if they experienced severe irAEs [10]. In a Japanese lung cancer cohort treated with nivolumab, patients with early irAEs showed improved ORR and PFS compared with those without [12]. However, these reports were retrospective in nature, similar to ours, and whether or not the presence of irAEs could be a novel predictor of response should be further validated in 
prospective trials. One possibility is that the longer exposure to nivolumab increases the risk of developing irAEs. Still, the association between irAEs and the efficacy of ICIs highlights the need for better management of irAEs so that patients can continue treatment as long as possible.

We noted that elderly patients demonstrated similar benefits compared to those in the overall population. Efficacy was similar among patients aged $<65,65$ to $<75, \geq 75$ years, and safety profiles were also similar. When age group was divided into $<75$, and $\geq 75$ years, patients who were aged $\geq$ 75 showed a significantly longer PFS, although this was no longer significant in Cox proportional hazard models. While elderly patients are often under-represented in clinical trials [13], recent real-world data suggests that the efficacy of ICIs does not deteriorate in elderly patients $[14,15]$. In a large French study, advanced NSCLC patients aged 80 years or over showed similar median OS compared to patients under 80 years, suggesting that no specific tolerability issue arose in this age group [15]. In an Italian EAP study, tumor response was similar across patients aged $<65,65$ to $<75$, and $\geq 75$ years [14]. Therefore, we cautiously suggest that old age alone should not be a barrier to anti-PD-1/PD-L1 treatment, but further study of larger elderly populations is warranted.

In our study, squamous histology seemed associated with higher ORR, although it did not lead to improved survival outcomes. This could be explained by the higher prevalence of oncogenic driver mutations in adenocarcinomas, which are reported to be less responsive to anti-PD-1/PD-L1 therapy $[16,17]$. Similarly, former or current smokers had a prolonged OS compared to never-smokers, and smoking status maintained significant after multivariate analysis. This is in line with a recent meta-analysis that PD-1 and PD-L1 inhibitors significantly prolonged the OS in smoking patients [18].

Liver metastasis was associated with poor survival outcome in our study. The presence of liver metastasis significantly increased the likelihood of death (hazard ratio, 2.18; $\mathrm{p}=0.001$ ) in multivariate analysis. A recent study which explored the association of liver metastasis and response in patients with melanoma and lung cancer also suggested that liver metastasis was associated with reduced response and shorter PFS [19]. In this study, reduced CD8 ${ }^{+} \mathrm{T}$ cell density at the invasive tumor margin was observed in liver biopsies, providing a possible background for poor survival. Multiple mechanisms have been suggested to explain liver-induced immune tolerance, such as poor CD4+ T cell activation [20], and Kupffer cells activating regulatory T cells [21]. Further mechanistic studies may aid to explain factors influencing response to ICIs.

The presence of EGFR mutation in tumor is known to be poorly responsive to ICIs. In a meta-analysis by Lee et al. [22], ICIs were not superior to docetaxel in EGFR-mutant subset, and in another meta-analysis, the PFS was in fact worse in patients with EGFR-mutant subset treated with PD-1/PD-
L1 inhibitors versus docetaxel [23]. In our study, ORR, PFS, and OS were not different according to EGFR mutation status, but this may be due to small number of EGFR-mutant patients and EGFR mutation status was largely unavailable in most patients.

Thirty-eight patients (12.7\%) with ECOG PS 2 were enrolled in our study, and efficacy results showed that ORR, PFS, and OS were not significantly inferior in ECOG PS 2 patients. This indicates that, unlike clinical trials, ECOG PS 2 patients can also benefit from ICIs in the real-world setting.

Our study has some limitations. The EAP did not require the PD-L1 status of tumor tissue for enrollment, so our data lacks analysis on the PD-L1 status and efficacy. Furthermore, there were no data on brain response evaluation to evaluate intracranial efficacy.

In conclusion, the efficacy of nivolumab in real-world patients seems to be comparable to that of clinical trials, and nivolumab is a viable option in the previously treated NSCLC patients.

\section{Electronic Supplementary Material}

Supplementary materials are available at Cancer Research and Treatment website (https://www.e-crt.org).

\section{Conflict of Interest}

Conflict of interest relevant to this article was not reported.

\section{Author Details}

${ }^{1}$ Division of Medical Oncology, Yonsei Cancer Center, Yonsei University College of Medicine, Seoul, ${ }^{2}$ Division of Oncology, Department of Internal Medicine, Asan Medical Center, University of Ulsan College of Medicine, Seoul, ${ }^{3}$ Division of Medical Oncology, Department of Internal Medicine, Seoul St. Mary's Hospital, College of Medicine, The Catholic University of Korea, Seoul, ${ }^{4}$ Division of Hematology-Oncology, Samsung Medical Center, Sungkyunkwan University School of Medicine, Seoul, ${ }^{5}$ Department of Internal Medicine, Seoul National University Hospital, Seoul, ${ }^{6}$ Department of Internal Medicine, Chonnam National University Hwasun Hospital, Hwasun, ${ }^{7}$ Center for Lung Cancer, National Cancer Center, Goyang, ${ }^{8}$ Division of Hematology and Medical Oncology, Department of Internal Medicine, Seoul National University Bundang Hospital, Seongnam, ${ }^{9}$ Department of Internal Medicine, Korea University Guro Hospital, Seoul, ${ }^{10}$ Department of Hematology/Oncology, Keimyung University Dongsan Hospital, Daegu, ${ }^{11}$ Division of Medical Oncology, Department of Internal Medicine, St. Vincent's Hospital, College of Medicine, The Catholic University of Korea, Suwon, ${ }^{12}$ Division of Oncology, Department of Internal Medicine, Gachon University Gil Medical Center, Incheon, ${ }^{13}$ Department of Internal Medicine, Kosin University Gospel Hospital, Busan, ${ }^{14}$ Department of Internal Medicine, Veterans Health Service Medical Center, Seoul, ${ }^{15}$ Department of Internal Medicine, CHA Bundang Medical Center, CHA University, Seongnam, ${ }^{16}$ Department of Hematology-Oncology, Inje University Haeundae Paik Hospital, 
Busan, ${ }^{17}$ Division of Hematology/Oncology, Department of Internal Medicine, Korea Cancer Center Hospital, Korea Institute of Radiological and Medical Sciences, Seoul, ${ }^{18}$ Department of Internal Medicine, Kyungpook National University Hospital, School of
Medicine, Kyungpook National University, Daegu, ${ }^{19}$ Division of Hematology-Oncology, Department of Internal Medicine, Chungbuk National University Hospital, Chungbuk National University College of Medicine, Cheongju, Korea

\section{References}

1. Taube JM, Klein A, Brahmer JR, Xu H, Pan X, Kim JH, et al. Association of PD-1, PD-1 ligands, and other features of the tumor immune microenvironment with response to antiPD-1 therapy. Clin Cancer Res. 2014;20:5064-74.

2. Borghaei H, Paz-Ares L, Horn L, Spigel DR, Steins M, Ready $\mathrm{NE}$, et al. Nivolumab versus docetaxel in advanced nonsquamous non-small-cell lung cancer. N Engl J Med. 2015;373:162739.

3. Brahmer J, Reckamp KL, Baas P, Crino L, Eberhardt WE, Poddubskaya E, et al. Nivolumab versus docetaxel in advanced squamous-cell non-small-cell lung cancer. N Engl J Med. 2015;373:123-35.

4. Gettinger S, Borghaei H, Brahmer J, Chow L, Burgio M, De Castro Carpeno J, et al. Five-year outcomes from the randomized, phase 3 trials CheckMate 017/057: nivolumab vs docetaxel in previously treated NSCLC. In: 2019 World Conference on Lung Cancer; 2019 Sep 7-10; Barcelona, Spain, Abstract No. OA14.04.

5. Lilenbaum RC, Cashy J, Hensing TA, Young S, Cella D. Prevalence of poor performance status in lung cancer patients: implications for research. J Thorac Oncol. 2008;3:125-9.

6. Sacco PC, Casaluce F, Sgambato A, Rossi A, Maione P, Palazzolo $G$, et al. Current challenges of lung cancer care in an aging population. Expert Rev Anticancer Ther. 2015;15:141929.

7. Eisenhauer EA, Therasse P, Bogaerts J, Schwartz LH, Sargent D, Ford R, et al. New response evaluation criteria in solid tumors: Revised RECIST guideline (version 1.1). Eur J Cancer. 2009;45:228-47.

8. Rogado J, Sanchez-Torres JM, Romero-Laorden N, Ballesteros AI, Pacheco-Barcia V, Ramos-Levi A, et al. Immune-related adverse events predict the therapeutic efficacy of anti-PD-1 antibodies in cancer patients. Eur J Cancer. 2019;109:21-7.

9. Sato K, Akamatsu H, Murakami E, Sasaki S, Kanai K, Hayata $\mathrm{A}$, et al. Correlation between immune-related adverse events and efficacy in non-small cell lung cancer treated with nivolumab. Lung Cancer. 2018;115:71-4.

10. Fujii T, Colen RR, Bilen MA, Hess KR, Hajjar J, Suarez-Almazor ME, et al. Incidence of immune-related adverse events and its association with treatment outcomes: the MD Anderson Cancer Center experience. Invest New Drugs. 2018;36:638-46.

11. Teraoka S, Fujimoto D, Morimoto T, Kawachi H, Ito M, Sato $\mathrm{Y}$, et al. Early immune-related adverse events and association with outcome in advanced non-small cell lung cancer patients treated with nivolumab: a prospective cohort study. J Thorac
Oncol. 2017;12:1798-805.

12. Haratani K, Hayashi H, Chiba Y, Kudo K, Yonesaka K, Kato $\mathrm{R}$, et al. Association of immune-related adverse events with nivolumab efficacy in non-small-cell lung cancer. JAMA Oncol. 2018;4:374-8.

13. Shenoy P, Harugeri A. Elderly patients' participation in clinical trials. Perspect Clin Res. 2015;6:184-9.

14. Grossi F, Crino L, Logroscino A, Canova S, Delmonte A, Melotti B, et al. Use of nivolumab in elderly patients with advanced squamous non-small-cell lung cancer: results from the Italian cohort of an expanded access programme. Eur J Cancer. 2018;100:126-34.

15. Assie JB, Cotte F, Levra MG, Calvet C, Jolivel R, Jouaneton $B$, et al. Nivolumab outcomes in octogenarian patients with advanced non-small cell lung cancer in a French real-world setting. J Thorac Oncol. 2019;14(10 Suppl):S708.

16. Soo RA, Lim SM, Syn NL, Teng R, Soong R, Mok TS, et al. Immune checkpoint inhibitors in epidermal growth factor receptor mutant non-small cell lung cancer: current controversies and future directions. Lung Cancer. 2018;115:12-20.

17. Sakamoto H, Tanaka H, Shiratori T, Baba K, Ishioka Y, Itoga $\mathrm{M}$, et al. The efficacy of immune checkpoint inhibitors in advanced non-small cell lung cancer harboring driver mutations. Mol Clin Oncol. 2019;10:610-4.

18. Li B, Huang X, Fu L. Impact of smoking on efficacy of PD-1/ PD-L1 inhibitors in non-small cell lung cancer patients: a meta-analysis. Onco Targets Ther. 2018;11:3691-6.

19. Tumeh PC, Hellmann MD, Hamid O, Tsai KK, Loo KL, Gubens MA, et al. Liver metastasis and treatment outcome with antiPD-1 monoclonal antibody in patients with melanoma and NSCLC. Cancer Immunol Res. 2017;5:417-24.

20. Wang JC, Livingstone AM. Cutting edge: CD4+ T cell help can be essential for primary CD8+ T cell responses in vivo. J Immunol. 2003;171:6339-43.

21. Jenne $C N$, Kubes P. Immune surveillance by the liver. Nat Immunol. 2013;14:996-1006.

22. Lee CK, Man J, Lord S, Links M, Gebski V, Mok T, et al. Checkpoint inhibitors in metastatic EGFR-mutated non-small cell lung cancer: a meta-analysis. J Thorac Oncol. 2017;12:403-7.

23. Dong ZY, Zhang JT, Liu SY, Su J, Zhang C, Xie Z, et al. EGFR mutation correlates with uninflamed phenotype and weak immunogenicity, causing impaired response to PD-1 blockade in non-small cell lung cancer. Oncoimmunology. 2017;6:e1356145. 